\title{
Micrometer measurements of double stars from the Spanish observatories at Calar Alto and Santiago de Compostela ${ }^{\star}$
}

\author{
J.A. Docobo \\ Observatorio Astronómico "Ramón María Aller", P.O. Box 197, Universidade de Santiago de Compostela, Spain \\ e-mail: oadoco@usc.es
}

Received October 28; accepted November 18, 1997

\begin{abstract}
This paper reports 458 micrometer observations of visual double stars made with the $152 \mathrm{~cm}$. telescope at Calar Alto Observatory (Almería, Spain) and with the $35 \mathrm{~cm}$. telescope at Ramón María Aller Observatory (Santiago de Compostela, Spain).
\end{abstract}

Key words: astrometry — stars: binaries: close — binaries: visual

\section{Introduction}

Almost $40 \%$ of the observations presented here were made with the $152 \mathrm{~cm}$. Spanish telescope at E.O.C.A., Calar Alto Observatory (Almería, Spain) during an observation run at the end of July 1995. The procedure was the same as for the earlier runs performed with this telescope and using the same micrometer, see Couteau et al. (1989) and Docobo \& Prieto (1993).

Together with these data, micrometer measurements made with the $35 \mathrm{~cm}$ reflector at Ramón María Aller Observatory (Santiago de Compostela, Spain) are included. In this latter case, the observations were carried out in 1994 and 1995 and to a lesser extent in 1996 and 1997.

Although the micrometers used at the two Observatories were different (both were made at Nice Observatory, France), the eyepieces were the same: 12, 10 and $8 \mathrm{~mm}(x 1017, x 1220, x 1525$ at Calar Alto and $x 325$, $x 390, x 488$ at Santiago de Compostela, respectively).

With respect to the small micrometer in the $35 \mathrm{~cm}$ telescope, its angular equivalent of the screw pitch was 23 "' 35 , i.e., 0 "' 467 per division. Using this telescope in good seeing conditions, a bright double star close to 0.5 can be separated.

Send offprint requests to: J.A. Docobo

* Tables 1 and 2 only available in electronic form at CDS via anonymous ftp to cdsarc.u-strasbg.fr (130.79.128.5) or via http://cdsweb.u-strasbg.fr/Abstract.html

\section{Observations}

The 458 micrometer measurements are listed in Tables 1 and 2. Table 1 includes 179 observations of 102 stars performed at Calar Alto Observatory and Table 2 includes 279 observations of 102 stars at Ramón María Aller Observatory. The results are presented in the same format in both Tables: each binary is identified by its WDS catalogue number (Worley \& Douglass 1996), followed by the star's name and its ADS catalogue number (Aitken 1932) if any. Letters denote the components in the case of the multiple star systems. Subsequent columns represent the observation epoch, the position angle, the angular separation together with the number of nights on which the star was observed. In no case did the measurements averaged differ by more than $2^{\circ}$ for position angles. For angular separation, the estimated error varies from $10 \%$ for the very close pairs to less than $1 \%$ for the very wide ones.

Acknowledgements. I would like to thank the Instituto Geográfico Nacional, the staff and assistant personnel at E.O.C.A. for their help and the facilities made available at Calar Alto. This work was carried out as part of the research projects PB 92-1074 and XUGA 24301B96 supported by D.G.I.C.Y.T. and the Xunta de Galicia respectively.

\section{References}

Aitken R.G., 1932, New General Catalogue of Double Stars (A.D.S.). Carnegie Institution of Washington No. 417

Couteau P., et al., 1989, A\&AS 78, 483-486

Dococbo J.A., Prieto C., 1993, A\&AS 100, 641-646

Worley C.E., Douglass G.G., 1996, The Washington Visual Double Star Catalogue, 1996.0 Electronic version Naval Observatory WWW (http:// aries.usno.navy.mil/ad/wds/.) 